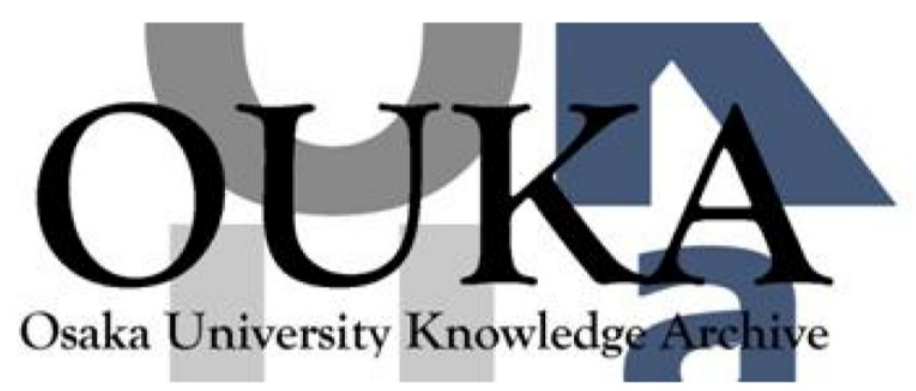

\begin{tabular}{|c|c|}
\hline Title & $\begin{array}{l}\text { Across-wafer nonuniformity of long throw } \\
\text { sputter deposition }\end{array}$ \\
\hline Author (s) & Mayo, A. A.; Hamaguchi, S.; Joo, J. H. et al. \\
\hline Citation & $\begin{array}{l}\text { Journal of Vacuum Science and Technology B: } \\
\text { Microelectronics and Nanometer Structures. } \\
\text { 15(5) p. } 1788-p .1793\end{array}$ \\
\hline Issue Date & $1997-09$ \\
\hline oaire:version & VoR \\
\hline URL & ht tps://hdl. hand le. net/11094/78498 \\
\hline rights & $\begin{array}{l}\text { This article may be downloaded for personal use } \\
\text { only. Any other use requires prior permission } \\
\text { of the author and AIP Publishing. This article } \\
\text { appeared in Journal of Vacum Science } \& \\
\text { Technology B: Microelectronics and Nanometer } \\
\text { Structures Processing, Measurement, and } \\
\text { Phenomena 15, 1788 (1997) and may be found at } \\
\text { https://doi.org/10.1116/1.589526. }\end{array}$ \\
\hline Note & \\
\hline
\end{tabular}

Osaka University Knowledge Archive : OUKA

https://ir. Library. osaka-u. ac. jp/

0saka University 


\title{
Across-wafer nonuniformity of long throw sputter deposition
}

\author{
A. A. Mayo and S. Hamaguchi \\ IBM T.J. Watson Research Center, Yorktown Heights, New York 10598 \\ J. H. JoO \\ Kunsan National University, Korea \\ S. M. Rossnagel ${ }^{\text {a) }}$ \\ IBM T.J. Watson Research Center, Yorktown Heights, New York 10598
}

(Received 23 January 1997; accepted 6 June 1997)

\begin{abstract}
Physical sputter deposition has been used at longer-than-normal cathode-to-sample distances for semi-directional deposition within high aspect ratio features. "Long throw" sputter deposition can be advantageous over other means of directional sputtering, such as collimated sputter deposition, because of the absence of collimators and related problems. However, due to the finite target size and sample geometry, an asymmetry is observed at the wafer edge with a thicker deposit on the inward-facing walls of trench and via structures compared with the outward-facing walls. We have used numerical simulation as well as metal sputter deposition experiments to characterize this asymmetry, which is typically $2-3: 1$ at the wafer edge. We also discuss how ionized sputter deposition would alter the deposition profile in the edge region'. (C) 1997 American Vacuum Society. [S0734-211X(97)00405-8]
\end{abstract}

\section{INTRODUCTION}

Magnetron sputtering, also know generically as physical sputtering or physical vapor deposition (PVD), is widely used to deposit metal layers on semiconductor wafers. The deposition process using magnetrons results in a very broad angular distribution of the depositing atoms, which is very useful for making planar films, but inappropriate for depositing films into deep structures. As the feature size on semiconductor wafers has reduced, the effective aspect ratio (depth/width of a feature) has increased. Using conventional magnetron sputter deposition in this case results in a rapid closing-off of the feature, and the formation of a buried void.

A broad number of deposition technologies have been attempted to overcome this fundamental problem. In the sputtering realm, the primary technologies have been collimated sputter deposition, ${ }^{1,2}$ elevated temperature/reflow deposition, ${ }^{3,4}$ long throw sputter deposition, ${ }^{5-8}$ and most recently ionized magnetron sputter deposition. ${ }^{9-11}$ The film deposition is complicated by the potential need for adhesion layers, diffusion barrier layers and seed layers, all of which should be primarily conformal within the feature as well as quite thin.

In present-day manufacturing applications, collimated sputter deposition is used for the deposition of contact and diffusion barrier layers within moderate aspect ratio (AR $=1-3$ ) features. However, collimated sputter deposition has been characterized by problems relating to the thick deposits formed on the collimator surfaces. These result in a gradual change in the uniformity profile of the tool, as well as in concerns about flaking and contamination. More importantly, collimated sputtering results in significant added cost to the film layer due to the intrinsic inefficiency of the collimated deposition process, as well as all of the other problems.

\footnotetext{
a)Electronic mail: rossn@watson.ibm.com
}

Long throw sputtering is intrinsically similar to collimated sputter deposition in that the depositing flux is filtered such that high angle sputtered atoms cannot reach the sample. In a long throw system, the sample is placed roughly 1 target diameter $(25 \mathrm{~cm})$ away from the cathode. Sputtered atoms which are not ejected from the cathode in a nearnormal angle of incidence are likely to deposit on the chamber walls rather than on the sample. In effect, the entire chamber becomes a single cell of a collimator. The deposition efficiency is not changed in this manner, but the thick deposits of non-normal-angle-of-incidence atoms are spread over a larger region of the wall and result in fewer maintenance issues compared to collimation. Effectively, though, long throw and collimation are similar technologies, with the added requirement for long throw being a much lower working gas pressure to reduce scattering.

Long throw system geometries are ideal on the wafer centerline, but have geometrical problems near the wafer edge. While the "planar" deposition rate at the wafer edge can be made uniform with the wafer centerline, it is very difficult to make the "directional" deposition process uniform. This is shown in Fig. 1, where the asymmetry in the depositing flux at the wafer edge is described. The limited size of the cathode, which is essential to any long throw system, means that the edge regions of the sample receive more deposition flux from the center than from the edge of the target. This will result in potentially thick deposits on the inward-facing walls of trenches and vias at the wafer edge compared with outward-facing walls. ${ }^{5,7,8}$

This effect will be strongly dependant on the aspect ratio of the feature as well as the exact radial location. The present study examines these geometrical issues as a function of aspect ratio for thin liner or diffusion barrier films. The goal is to quantify the degree of the asymmetry and identify whether any other factors are present, such as a less-than-unity stick- 


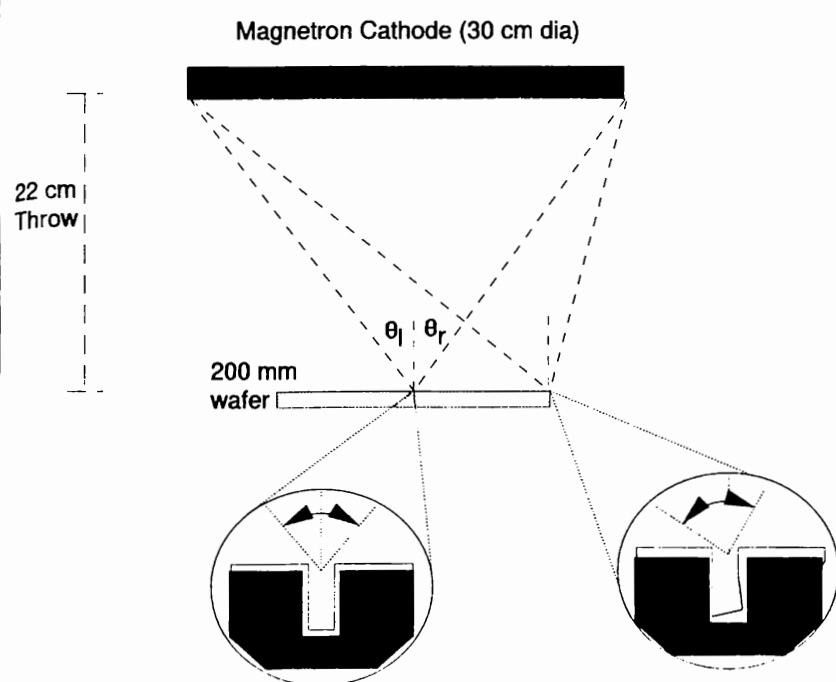

FIG. 1. Orientation of the cathode and wafer sample in a long throw deposition system, drawn to scale. The two angles, THETA-sub-l and THETAsub-r, relate to the maximum angular divergence of the allowed trajectories of the depositing atoms.

ing coefficient, that may alter the profile. The study compares experimental results from a conventional, commercial sputter deposition tool with the results of numerical simulation of the deposition process. This approach has been used in related studies on the effects of partial ionization on the deposition process. ${ }^{11,12}$ The present study also briefly examines the effect of an ionized deposition technology (I-PVD) on this fundamental asymmetry.

\section{SIMULATION}

In order to model the sputter metal deposition process we have used the simulator shock tracking algorithm for deposition and etching (SHAPE). This is a two dimensional code in which a shock tracking algorithm is used for the surface evolution. ${ }^{13}$ It can be used whether or not ionization and etching are present. In the code the surface is modeled by a sequence of nodes, and the deposition and etch rates are calculated at each node.

To determine the deposition rate and the etch rate when there is any etching we assume that the flow and diffusion of material along the surface are negligible. This is a reasonable assumption for most metals on a low temperature substrate. Since the length scales of the trenches (microns) are sufficiently smaller than the mean-free paths of the particles $(\mathrm{cm})$, we assume collisionless transport within the surface feature. We also assume that any metal atoms sputtered by incident ions have low enough energy, so that they do not resputter the surface when they reach other surface sites. Instead, we assume that these atoms and the neutral metal atoms from the plasma source are adsorbed on the surface with constant sticking coefficient $S$. Re-emitted atoms from the surface are assumed to have the cosine angular distribution about the surface normal, and if a high energy ion hits the wafer it sputters the surface with sputtering yield that only depends on the angle formed by the surface normal and the direction of the ion.

We can derive expressions for all the fluxes. We first note that the normal component of the outgoing flux at position $X$ on the surface is the sum of the desorption flux, that is the flux of re-emitted (neutral) metal atoms, and the resputtering flux $F^{\text {etch }}$ :

$$
F^{\text {out }}(X)=(1-S) F^{\text {in }}(X)+F^{\text {etch }}(X) .
$$

Here $F^{\text {in }}$ is the incoming flux of low energy metal atoms, and so $(1-S) F^{\text {in }}$ represents the total desorption flux $F^{R}$.

The incoming neutral flux is the sum of the incoming neutral metal flux $F^{R}$ that is re-emitted from other points on the surface, and the metal-atom flux $F^{D}$ that arrives at the surface directly from the magnetron cathode:

$$
F^{\text {in }}(X)=F^{R}(X)+F^{D}(X) .
$$

Using these equations and our assumption about the velocity distribution of the re-emitted atoms we can derive an integral equation for the desorption flux:

$$
F^{R}(X)=(1-S) \int d s F^{R} K_{\nu}(X, w)+G(x) .
$$

The kernel $K_{\nu}(X, w)$ depends on the surface geometry. In particular, its computation involves the evaluation of a visibility factor $g$ (i.e., $g=1$ if the points $X$ and $w$ are on the line of sight, and $g=0$ otherwise). Its evaluation requires $O\left(n^{2}\right)$ operations where $n$ is the number of nodes on the surface.

We calculate the flux $G(x)$ from the distribution functions for metal ions, inert gas ions, and the metal atoms that are directly emitted from the magnetron target. Once we have evaluated $G$ we solve the integral equation to determine the flux $F^{R}$ of the re-emitted metal atoms. For details, see Ref. 14.

To determine the neutral metal flux $F^{D}(X)$ emitted directly from the magnetron cathode, we note that it can be expressed in terms of the distribution function of the sputtered metal ions on the target cathode $f^{D}(v)$ :

$$
\begin{aligned}
F^{D}(X)= & \frac{1}{\pi} \int_{\theta_{l}}^{\theta_{r}} d \theta \int_{0}^{\pi} d \phi \int_{0}^{\infty}\left(N_{x} \sin ^{2} \theta \cos \psi\right. \\
& \left.+N_{z} \sin \theta \cos \theta\right) v^{3} f^{D}(v) .
\end{aligned}
$$

Here $\theta_{l}$ and $\theta_{r}$ are the left and right collimation angles, $\theta$ and $\phi$ are the usual polar angles around the $z$ direction, and $N=\left(N_{x}, 0, N_{z}\right)$ is the surface normal.

If the sample is horizontal and flat, the neutral flux is independent of $X$, and given by

$$
G\left(\theta_{l}, \theta_{r}\right)=\frac{\pi}{2}\left(\cos 2 \theta_{r}-\cos 2 \theta_{l}\right) \int d v \dot{v}^{3} f^{D}(v) .
$$

Using this expression, the flux at a general point can be expressed 


$$
\begin{aligned}
F^{D}(X)= & \frac{2 G\left(\theta_{l}, \theta_{r}\right)}{\cos 2 \theta_{r}-\cos 2 \theta_{l}} \int_{\theta_{l}}^{\theta_{r}} d \theta \int d \psi\left(N_{x} \sin ^{2} \theta \cos \psi\right. \\
& \left.+N_{z} \sin \theta \cos \theta\right) .
\end{aligned}
$$

We assume that the distribution function $f^{D}$ is isotropic, i.e., independent of the direction of $v$ and position $x$ on the target.

We can also calculate the etch rate $C_{m^{+}}$and metal deposition rate $D_{m}+$ from the prescribed metal ion distribution function. Precise formulas can be found in Refs. 10 and 12 .

Given $F^{R}$ and $F^{D}$ we compute the deposition rates $D^{R}=(m S / \rho) F^{R}$ and $D^{D}=(m S / \rho) F^{D}$, where $m$ is the mass of a metal ion, and $\rho$ is the mass density of the metal film on the surface, and then rate of metal deposition

$$
D=D^{R}+D^{D}+D_{m}+\text {. }
$$

The net velocity of the surface is given by $C=C_{m^{+}}-D$.

\section{EXPERIMENTAL AND NUMERICAL RESULTS}

Thin Ta films were sputter deposited onto patterned wafer samples using a conventional rotating magnet magnetron of diameter $30 \mathrm{~cm}$. Samples, which consisted of arrays of trenches and vias, were configured at a fixed throw distance and arranged radially from the system centerline. The operating pressure during film deposition was $0.6-0.8$ mTorr of $\mathrm{Ar}$, the cathode was operated at $2.5 \mathrm{~kW} \mathrm{dc}$, and the deposition time was adjusted to deposit a film of $100 \mathrm{~nm}$ thickness on the "field" or flat areas of the samples.

The spacing between the cathode and the sample chuck was fixed in this study at $22 \mathrm{~cm}$. This is consistent with earlier work ${ }^{15}$ which showed good conformality of the deposited films on the centerline of the system at this throw distance. The transport of sputtered $\mathrm{Ta}$ in $\mathrm{Ar}$ at $0.8 \mathrm{mTorr}$ is assumed to be essentially ballistic (i.e., a small number of in-flight collisions or no collisions), in that the mean-free path for Ta-Ar collisions should exceed $12 \mathrm{~cm}$ and the collisional process for energetic $(25 \mathrm{eV}) \mathrm{Ta}$ with the much lighter, cold Ar should not result in significant directional scattering. The assumption of collision-free transport may be less valid for such materials as $\mathrm{Ti}$ or $\mathrm{Al}$, which have a much lower mass and a slightly lower sputtered kinetic energy.

Samples were located radially on the sample plane at near the sample/cathode centerline, and then in approximately 5 $\mathrm{cm}$ increments from the center. This results in three samples 1-3 which are consistent with the location of a $200 \mathrm{~mm}$ wafer (center, middle, and edge), a sample consistent with the cathode edge 4 and a sample several centimeters beyond the cathode edge 5 . These outer samples were intended mostly as a test of the modeling process, rather than to simulate actual deposition onto a wafer sample. The angular divergence of the depositing flux at each position is shown in Table I for the five locations used.

Figures 2(a) and 2(b) are scanning electron microcsopy (SEM) pictures of trench structures which are configured at the wafer edge position. Figure 2(a) was a trench oriented in the radial direction which was cleaved in the tangential direction. This structure shows no evidence of asymmetric
TABLE I. Angular divergences of depositing Ta atoms as a function of sample position. Normal incidence is $0^{\circ}$.

\begin{tabular}{ccc}
\hline \hline Sample & $\begin{array}{c}\text { Radial position } \\
(\mathrm{cm})\end{array}$ & $\begin{array}{c}\text { Flux arrival angles } \\
(+ \text { to }- \text { degrees })\end{array}$ \\
\hline$\{1\}$ & 2 & +29 to -34 \\
$\{2\}$ & 6 & +21 to -40 \\
$\{3\}$ & 10 & +11 to -46 \\
$\{4\}$ & 14 & +2 to -51 \\
$\{5\}$ & 20 & -15 to -58 \\
\hline \hline
\end{tabular}

deposition. Figure 2(b) is a nearby feature which was oriented tangentially and cleaved radially. This shows the strong asymmetry which can be present in the deposit, comparing the inward- and outward-facing sidewalls.

The step coverage, or relative thickness at a specific location on the sample compared to the top field coverage, was measured for a wide variety of features with aspect ratios of from 0.5 to 4.5 . The step coverage was measured by means of SEM cross sections of cleaved, unpolished samples. The physical orientation of each wafer sample was known, and the step coverage was measured on the walls of separate trenches which were aligned radially and tangentially at the same radial position. In the case of radially oriented trenches, no side-to-side asymmetries were observed. For the tangentially oriented trenches, strong asymmetries were observed comparing the trench walls which faced the centerline of the system (inward-facing), compared to the opposing sidewall which faced the chamber wall (outward-facing).

Figure 3 shows the measured step coverage for trench sidewalls, at the bottom corner for both inward-facing and outward-facing sidewalls as a function of the aspect ratio of the trench. In general, the step coverage for a trench sidewall would be expected to scale as $1 /(1+2 \mathrm{AR})$ where AR is the aspect ratio. This is due simply to the supply of available atoms to the trench. For example, a trench of aspect ratio 2:1 should not have a step coverage greater than $20 \%$, if the atoms are uniformly distributed (conformally) on the trench walls and bottom.

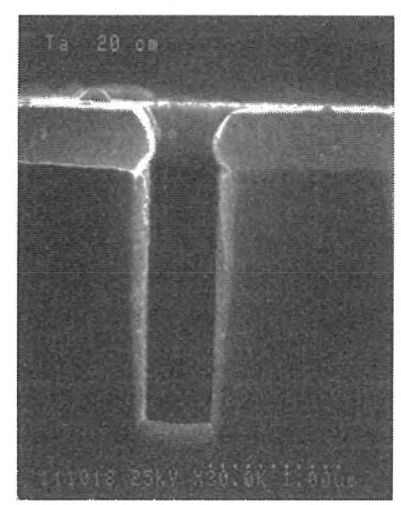

(a)

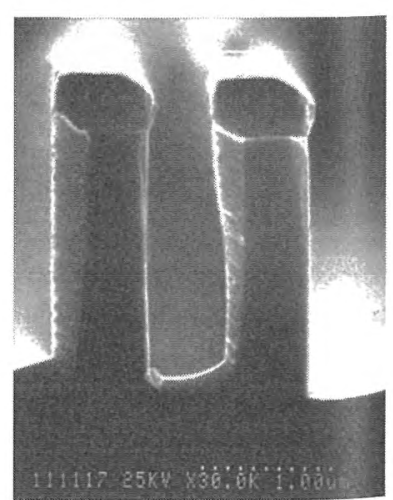

(b)
FIG. 2. SEM cross sections of moderate aspect ratio features deposited at the wafer-edge position. (a) A via cleaved in the direction tangent to the wafer edge, (b) a trench cleaved in the radial direction. In (b) the centerline of the wafer is to the left. 


\section{Step Coverage at bottom corner}

\section{Step Coverage}

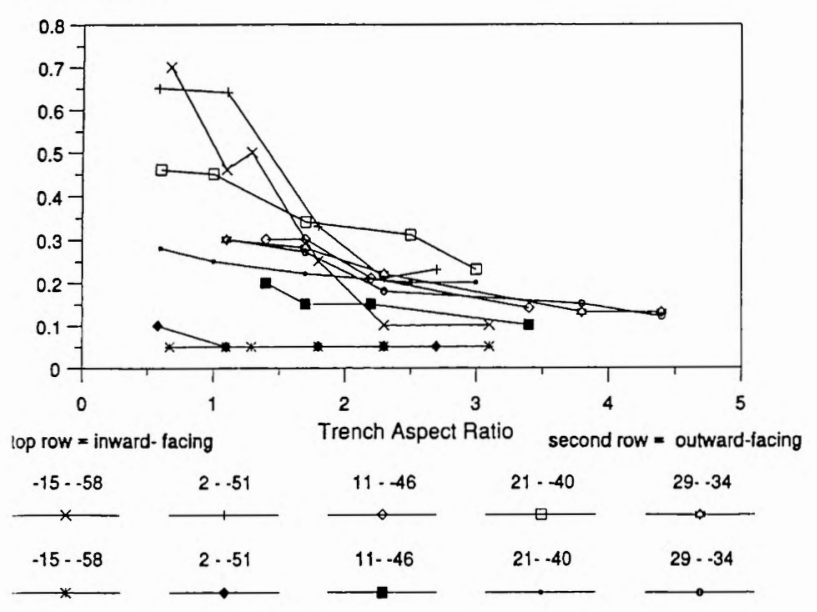

FIci. 3. The experimentally measured relative thickness, or "step coverage" at the extreme bottom corner of the trenches as a function of wafer position. In the key, the numbers refer to the allowed arrival angles for the depositing atoms. The top row is for trench walls which face inward towards the center of the wafer. The second row is for outward-facing trenches. The first column (in the key) correlates with wafer position 5 , the second column with position 4 , and so on.

In Fig. 3, samples 4 and 5 which were at or beyond the edge of the cathode show essentially no deposition $(<100$ A) on the outward-facing bottom corner. At positions consistent with a $200 \mathrm{~mm}$ wafer sample, the centermost position shows no asymmetries. The position $6 \mathrm{~cm}$ from the center and the position at the wafer edge both show a 2:1 ratio of inward- to outward-facing thickness which is only slightly dependent on aspect ratio.

The numerical model, SHADE, was then used to replicate the same deposition conditions that were present with the experimental samples. The simulations require several assumptions which may limit the exact match with the experiments. In particular, the model uses a clamped, isotropic flux distribution for the neutral deposition. The angular limits to the deposition flux were chosen to be similar to the experimental situation. Another constraint of the model is that it does not allow for an angular dependance to the sticking coefficient, which might be expected physically, and also it treats re-emission isotropically rather than specularly. And finally, there is no provision for surface effects such as diffusion or recrystallization.

As an example of the comparison between experiment and simulation, Figs. 4(a) and 4(b) show a trench of aspect ratio 2.5 which was deposited at the farthest-out position (sample position 5). The angular distribution of the flux arriving to this trench is between -15 and $-58^{\circ}$, which in the scope of Fig. 4 is coming from the left-hand side of the Fig. 4. Higher resolution measurements of Fig. 4(a) show some degree of deposition on the left (outer-facing) sidewall, which implies a nonunity sticking coefficient for the films on the right-hand, inward-facing sidewall. The results of the numerical simulation are shown in Fig. 4(b) for an identical

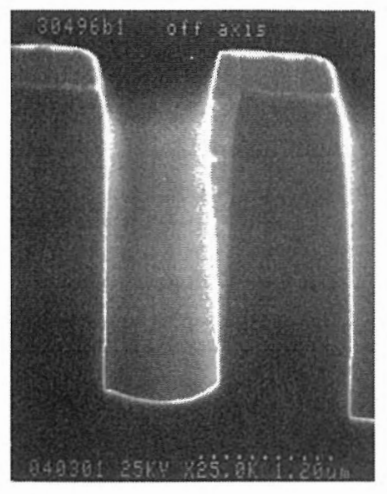

(a)

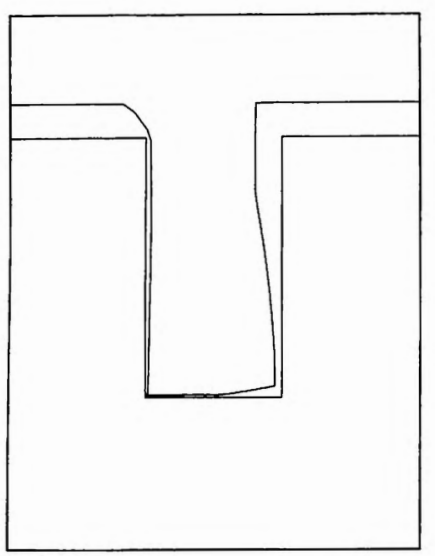

(b)
FIG. 4. (a) SEM micrograph and (b) simulation for the deposition at position 5 , which has an arrival angle for the depositing atoms from -15 to $-58^{\circ}$. The system centerline is to the far left of the figure.

deposition divergence coupled with an average sticking coefficient of 0.6. This value was found in earlier work ${ }^{15}$ to be consistent with the conformality observed on the system centerline. Figure 4(b) shows a similar overall profile to Fig. 4(a). It should be noted that this is a neutral-only deposition and has no resputtering of the deposited film.

Figures 5(a) and 5(b) show a similar situation for deposition at the radius of the cathode edge (sample position 4 ). The flux divergence angles in this case are +2 to $-51^{\circ}$, and in this case the center of the system is to the right-hand side of Fig. 5. In the simulation results [Fig. 5(b)] a sticking coefficient of 0.6 showed the best match between experiment and simulation.

Figures 6(a) and 6(b) show the case of the wafer-edge position (sample position 3), in which the arriving flux ranges from 11 to $-46^{\circ}$. Similar to Fig. 5 , the centerline of the system is to the right-hand side of Fig. 5. This case also uses an effective sticking coefficient of 0.6 . The exact shape

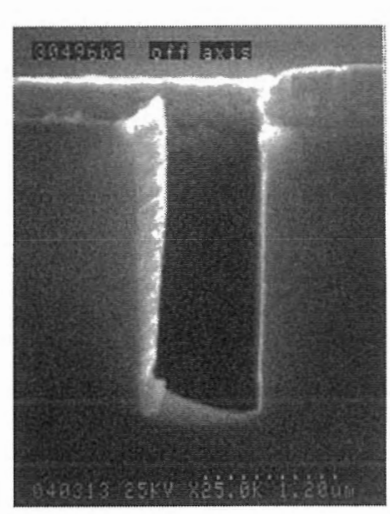

(a)

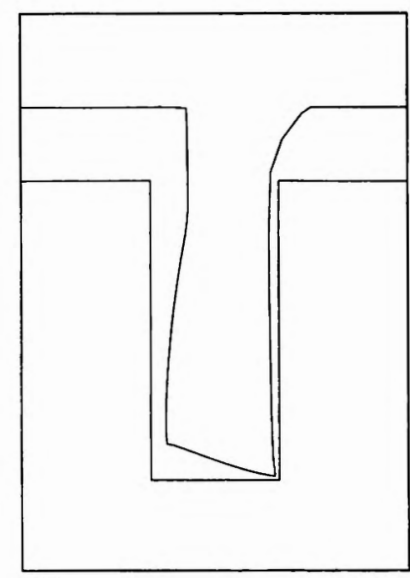

(b)
FIG. 5. (a) SEM micrograph and (b) simulation for the deposition at position 4 , which has an arrival angle for the depositing atoms of from +2 to $-51^{\circ}$. The system centerline is to the far right of this figure. 


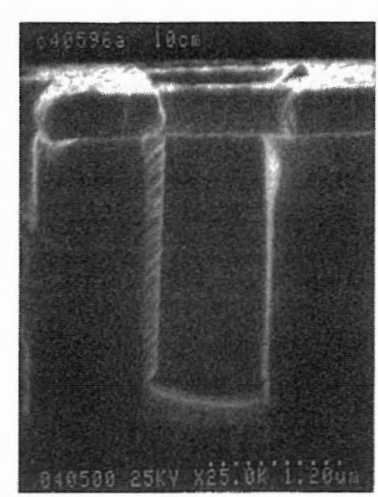

(a)

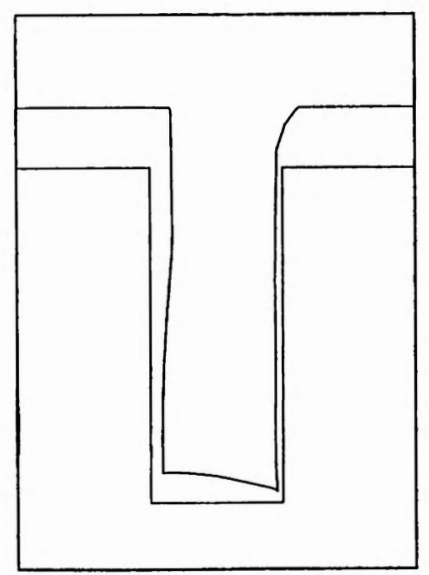

(b)
FIG. 6. SEM micrograph and (b) simulation for the case of deposition at the wafer edge (position 3), with an arriving angular flux from +11 to $-46^{\circ}$. The system centerline is to the right of this figure.

of the inward-facing sidewall deposition correlates in general with the simulation [Fig. 6(b)] but shows a more general taper than the more abrupt fall-off of the simulation.

\section{DISCUSSION}

The long throw deposition process relies on distance to eliminate the nonnormal incidence depositing atoms. In doing so, it eliminates the need for physical collimation and the problems which are associated with interposed collimators in a deposition system. However, long throw deposition is clearly asymmetric compared to collimated sputter deposition at positions away from the wafer centerline, and this asymmetry is typically $2-3: 1$ at the bottom corners of deep features and much higher $(6: 1)$ at the top corner of the feature. The current study used Ta as the depositing species. Tantalum is a desirable material for use with a $\mathrm{Cu}$-based interconnect metallization, but it also has a less-than-unity sticking coefficient due to its large kinetic energy and momentum. ${ }^{15}$ Titanium and $\mathrm{TiN}$, which are appropriate for $\mathrm{AlCu}$-based metallization, will have slightly different reflection and absorption dynamics due to the lighter atomic mass and reduced kinetic energy (compared to Ta). The functional result will be an increase in the in-flight scattering and a reduction of the geometrical directionality of the depositing flux. This will be significant for high aspect ratio features, but was not examined in this work.

It is not clear that there is any good remedy to the fundamental asymmetry of long throw deposition. An increase in the target diameter is functionally equivalent to a reduction in the throw distance, which results in less net directionality and lower step coverage at the bottom of features. Conversely, an increase in the throw distance (ultralong throw) to several cathode diameters results in a more uniform directionality across the wafer, but places extreme requirements on the operating gas pressure due to in-flight scattering. Current long throw tools operate for the most part without en-

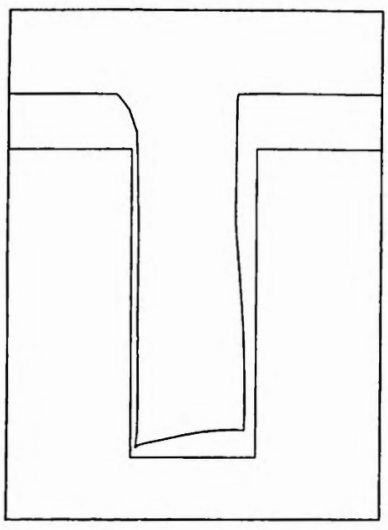

(a)

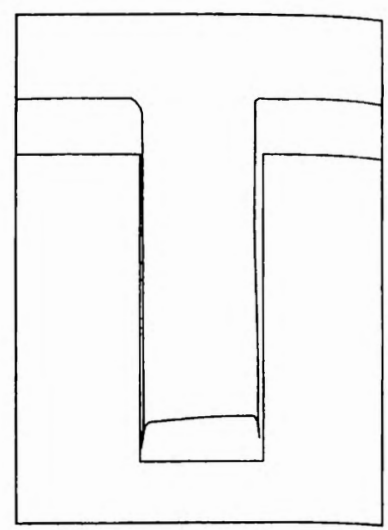

(b)
FIG. 7. Comparison of (a) conventional PVD deposition at the wafer edge 3 [similar to Figs. 6(a) and 6(b)] an I-PVD deposition with 50 the same position. The self-sputter yield for the depositing atoms used for (b) was 0.1 , consistent with a low depositing ion energy.

hancement, but further reductions in operating pressure (below 0.5 mTorr) would require an additional electron source, such as a hollow cathode. 6

The application of long throw sputtering is then one of the level of acceptable asymmetry. For modest aspect ratios (1:1) and thin films ( $<0.1$ of feature width), the $2: 1$ asymmetry may be acceptable. For higher aspect ratios or feature sizes below $0.5 \mu \mathrm{m}$, the asymmetry at the top of the feature is severe enough that to deposit a continuous, functional film on the outward facing sidewall will result in an unacceptable build-out of the inward facing wall.

A potential solution to the concern with asymmetric deposition characteristic of a long throw deposition is to partially ionize the depositing flux using a technique known as I-PVD. In this technique, the depositing flux passes through a dense inert gas plasma, and some fraction of the metal atoms are ionized. If a small bias voltage is then imposed on the sample, the metal ions from the plasma will be accelerated at normal incidence to the sample surface. This technique can be introduced to the SHADE model ${ }^{11,12}$ and now allows predictions of the degree of asymmetry of a deposition which consists of a nonsymmetric neutral flux coupled with a normal-incidence ionized flux.

The results of this simulation are shown in Figs. 7(a) and 7(b), where Fig. 7(a) is similar to Figs. 6(a) and 6(b), and is a neutral-only deposition. The neutral flux in this deposition was varied between +11 and $-46^{\circ}$, consistent with a location at the edge of a $200 \mathrm{~mm}$ wafer. In Fig. 7(b), the results are shown of an partially ionized deposition at the same location (50\% ionization), consistent with experimentally observed ionization levels and a very small resputter yield of 0.1 , which is consistent with sample bias voltages of several tens of volts dc.

There are several apparent features in the I-PVD deposition [Fig. 7(b)]. First, the bottom coverage is significantly higher, showing an average step coverage of $75 \%$. Second, the sidewall thicknesses are much thinner on both sides compared to the non-ionized deposition. This is consistent with 
the increased bottom-surface step coverage. Both of these effects are due to the near-normal incidence of the depositing metal ions, which make up 50\% of the depositing flux. In addition, the thickness of the inward-facing sidewall (in this case, the left sidewall) is reduced from about $3 \times$ the outward facing thickness at the top of the sidewall to about $2 \times$, and the tapering of the inward-facing thickness is less pronounced.

'S. M. Rossnagel, D. Mikalsen, H. Kinoshita, and J. Cuomo, J. Vac. Sci. Technol. A 9, 261 (1991).

${ }^{2} \mathrm{R}$. Joshi and S. Brodsky, Proceedings of the VLSI Multilevel Interconnection Conference, Santa Clara, CA, 1992, pp. 253-259.

'S. Ogawa, H. Nishamura, T. Kouzaki, and R. Sinclair, in Advanced Metallization for ULSI Teclinologies, edited by T. S. Cale and F. S. Pintchovski (Materials Research Society, Pittsburgh, 1992), p. 345.

${ }^{4} \mathrm{~K}$. Ngan, R. Mosely, Z. Xu, and I. Raaijmakers, Proceedings of the VLSI Multilevel Interconnection Conference, Santa Clara, CA, 1994, p. 452.

${ }^{5} \mathrm{~J}$. Broughton, C. Backhouse, M. Brett, S. Dew, and G. Este, Proceedings of the VLSI Multilevel Interconnection Conference, Santa Clara, CA, 1995, p. 201.

${ }^{6} J$. Cuomo and S. Rossnagel, J. Vac. Sci. Technol. A 4, 393 (1986).

${ }^{7}$ I. Wagner, in Proceedings of the VLSI Multilevel Interconnection Conference (VMIC), Santa Clara, CA, 1995, p. 226.

${ }^{8}$ T. Smy, K. Sheergar, S. K. Dew, and M. J. Brett, Proceedings of the VLSI Multilevel Interconnection Conference (VMIC), Santa Clara, CA, 1995, p. 670.

${ }^{9}$ S. M. Rossnagel and J. Hopwood, Appl. Phys. Lett. 63, 3285 (1993); J. Vac. Sci. Technol. B 12, 449 (1994).

${ }^{10}$ S. M. Rossnagel, Semiconductor International, Feb. 1996, p. 99.

${ }^{11}$ S. Hamaguchi and S. M. Rossnagel, J. Vac. Sci. Technol. B 13, 183 (1995).

${ }^{12}$ S. Hamaguchi and S. M. Rossnagel, J. Vac. Sci. Technol. B 14, 2603 (1996).

${ }^{13}$ S. Hamaguchi, M. Dalvie, R. T. Farouki, and S. Sethuraman, J. Appl. Phys. 74, 5172 (1993).

${ }^{14}$ S. Hamaguchi, Thin Solid Films 22, 81 (1996).

${ }^{15}$ S. M. Rossnagel, C. Nichols, S. Hamaguchi, R. Ruzic, and R. Turkot, J. Vac. Sci. Technol. B 14, 1819 (1996). 